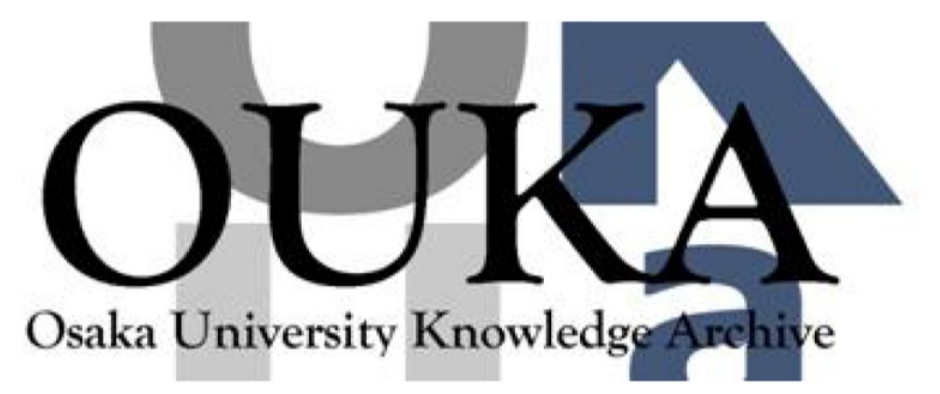

\begin{tabular}{|c|l|}
\hline Title & $\begin{array}{l}\text { Transport study of intense-laser-produced fast } \\
\text { electrons in solid targets with a preplasma } \\
\text { created by a long pulse laser }\end{array}$ \\
\hline Author(s) & Yabuuchi, T.; Paradkar, B.S. ; Wei, M.S. et al. \\
\hline Citation & Physics of Plasmas. 17(6) p. 060704 \\
\hline Issue Date & $2010-07$ \\
\hline oaire:version & VoR \\
\hline URL & https://hdl. handle. net/11094/3061 \\
\hline rights & \\
\hline Note & \\
\hline
\end{tabular}

Osaka University Knowledge Archive : OUKA

https://ir. Library. osaka-u. ac. jp/

Osaka University 


\title{
Transport study of intense-laser-produced fast electrons in solid targets with a preplasma created by a long pulse laser
}

\author{
T. Yabuuchi, ${ }^{1}$ B. S. Paradkar, ${ }^{1}$ M. S. Wei,${ }^{1}$ J. A. King, ${ }^{1}$ F. N. Beg, ${ }^{1}$ R. B. Stephens, ${ }^{2}$ \\ N. Nakanii, ${ }^{3}$ M. Hatakeyama, ${ }^{3}$ H. Habara, ${ }^{3}$ K. Mima, ${ }^{3}$ K. A. Tanaka, ${ }^{3}$ and J. T. Larsen ${ }^{4}$ \\ ${ }^{1}$ Center for Energy Research, University of California-San Diego, \\ La Jolla, California 92093, USA \\ ${ }^{2}$ General Atomics, San Diego, California 92186, USA \\ ${ }^{3}$ Graduate School of Engineering, Institute of Laser Engineering, Osaka University, \\ Yamada-oka, Suita, Osaka 565-0871, Japan \\ ${ }^{4}$ Cascade Applied Sciences, Inc., Longmont, Colorado 80503, USA
}

(Received 15 February 2010; accepted 18 May 2010; published online 29 June 2010)

\begin{abstract}
The effect of preplasma on fast electron generation and transport has been studied using an intense-laser pulse $\left(I=2 \times 10^{18} \mathrm{~W} / \mathrm{cm}^{2}\right)$ at the Osaka University. An external long pulse laser beam $(E<1.5 \mathrm{~J})$ was used to create various levels of preplasmas in front of a planar target for a systematic study. $K \alpha \mathrm{x}$-ray emission from a fluorescence layer (copper) was absolutely counted and its spatial distribution was monitored. Experimental data show $K \alpha$ x-ray signal reduction (up to $60 \%$ ) with an increase in the preplasma level. In addition, a ring structure of $K \alpha \mathrm{x}$ rays was observed with a large preplasma. The underlying physics of the ring structure production was studied by integrating the modeling using a radiation hydrodynamics code and a hybrid particle-in-cell code. Modeling shows that the ring structure is due to the thermoelectric magnetic field excited by the long pulse laser irradiation and an electrostatic field due to the fast electrons in the preplasma.

(C) 2010 American Institute of Physics. [doi:10.1063/1.3447878]
\end{abstract}

Fast ignition (FI) inertial confinement fusion is a relatively new concept and offers high gains compared to the central hot spot concept. ${ }^{1}$ It has separate compression and heating phases, which sets relatively less stringent requirements on the symmetric compression of the fuel. In advanced FI, a gold cone is inserted into the capsule to provide a clear path for the short pulse laser to deposit its energy near the compressed fuel. ${ }^{2}$ An important question that needs to be addressed is the preplasma effect on the laser to fast electron conversion and transport. This preplasma is produced by the inherent prepulse or amplified stimulated emission (ASE) in a laser system. The preplasma effect will be important when high energy lasers of 10-100 kJ are used for FI, where the Joule-level prepulse and ASE can create a large scale preplasma inside the cone. In addition, preplasma can also be created by preheating of the cone walls by $\mathrm{x}$ rays during the implosion. Furthermore, the high pressure of the implosion can break the cone tip, producing a jet of preplasma. The characteristics of the fast electrons (temperature, divergence, and number) can be changed due to laser self-focusing or filamantation in the preplasma. ${ }^{3-5}$ The electrostatic and magnetic fields ${ }^{6}$ in the preplasma can affect the fast electron transport. The effect of a preplasma inside the cone is of particular importance; however the cone's closed geometry causes difficulty in diagnosing the scale length and density profile of such a preplasma.

In this letter, effects of a large preplasma on the fast electron generation and transport have been systematically investigated using planar multilayer targets at the laser intensity of the order of $10^{18} \mathrm{~W} / \mathrm{cm}^{2}$ (this intensity is two orders of magnitude smaller than what is envisioned for the full scale FI, but is similar to that used in recent integrated experiments). ${ }^{7}$ The preplasma was created using an external long pulse laser beam. The preplasma density profiles were monitored using laser interferometry. Information about fast electrons was obtained by measuring the $K \alpha$ x rays emitted from a fluorescence layer buried in the targets. Experimental data show a decrease in $K \alpha$ signal up to $60 \%$ with an increase in preplasma level. In addition, a ring structure was observed in the $K \alpha$ images only when the long pulse laser beam was used. Integrated radiation hydrodynamics and hybrid particle-in-cell (PIC) modeling shows that the structure is due to electron deflections by a thermoelectric magnetic field excited by the long pulse laser irradiation and an electrostatic field due to the fast electrons in the preplasma.

The experiment was performed at the GMII laser facility at the Institute of Laser Engineering, Osaka University. The output laser pulse from an optical parametric chirped-pulse amplification system is split into two beams and then amplified independently. A laser pulse with energy of $12 \mathrm{~J}$ was delivered to the target after the pulse was compressed to $400-600$ fs by a segmented-grating pulse compression system. ${ }^{8}$ The short pulse was focused onto the target with a spot size of $20-30 \mu \mathrm{m}$ in full width at half maximum by a $f / 3.8$ off-axis parabolic mirror in $p$-polarization with an incident angle of $25^{\circ}$. The short pulse peak intensity was about $2 \times 10^{18} \mathrm{~W} / \mathrm{cm}^{2}$. Another chirped pulse with a $500 \mathrm{ps}$ duration was focused on the target via a $f / 13$ planoconvex lens with a maximum energy of $1.5 \mathrm{~J}$ to create plasma in advance of the short pulse irradiation. The long pulse beam spot was $200 \mu \mathrm{m}$ in diameter so that a quasi-onedimensional plasma expansion was encountered by the incident short pulse beam. The peak-to-peak time separation of the two pulses was 260 ps. Both pulses have a central wave- 


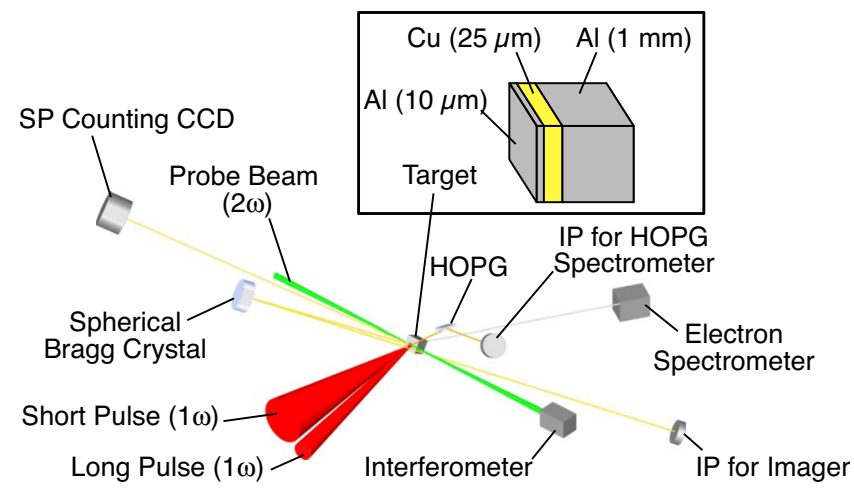

FIG. 1. (Color online) Schematic of the experimental setup. Details of target configuration are shown in the inset.

length of $1.053 \mu \mathrm{m}$. The density profile of the plasma was measured using interferometry technique with an optical probe $(\lambda=0.527 \mu \mathrm{m})$ of 50 ps before the short pulse interaction. Here, the probe beam is a pulse compressed down to 7 ps duration after it is partially clipped from the long pulse beam line. All three laser pulses originate from one laser pulse; therefore there is no timing jitter between these pulses.

Targets used in the experiment consist of aluminum and copper layers with an area of $1 \mathrm{~mm}$ square, as shown in the inset of Fig. 1. The laser pulses interact with the front $\mathrm{Al}$ layer (10 $\mu \mathrm{m}$ thick). This layer works as an ablator for the long pulse target interactions. The second layer is $25 \mu \mathrm{m}$ $\mathrm{Cu}$, which is the fluorescence layer emitting $K \alpha \mathrm{x}$ rays induced by the fast electrons. The target has another Al layer with a thickness of $1 \mathrm{~mm}$ on rear side. This thick Al layer minimizes the fast electron refluxing that makes the analysis difficult due to multiple passes of the fast electron through the fluorescence layer.

Several x-ray diagnostics were employed including the single photon (SP) counting technique with a charge coupled device (CCD) camera, an X-ray spectrometer with a highly oriented pyrolytic graphite (HOPG) crystal, and a twodimensional (2D) imaging system with a spherical quartz (2131) Bragg crystal. $K \alpha$ x-ray diagnostics are extremely useful for extracting information about fast electron transport. As fast electrons move into the target, they make binary collisions with $\mathrm{Cu}$ atoms, producing $K \alpha$ photons $(8.05 \mathrm{keV})$. There is a direct correspondence between the number of $K \alpha$ photons and the number of fast electrons since the cross section to produce $K \alpha$ photons by electron impact is quasiconstant with energy over $\sim 25 \mathrm{keV} .{ }^{9}$ These three diagnostics observed $\mathrm{Cu} K \alpha$ x-ray emission from the front of the target (laser-irradiated side), as shown in Fig. 1. The HOPG spectrometer was cross calibrated with the SP counting camera during the experiment; therefore, the absolute numbers of $K \alpha$ photons were obtained for all the data shots with a calibration uncertainty of $20 \%$. The crystal imager had a narrow bandwidth $(\sim 4 \mathrm{eV})$ at around the $\mathrm{Cu} K \alpha$ x-ray energy. Its magnification was $\sim 6$ and the spatial resolution was $22 \mu \mathrm{m}$. In addition to the $K \alpha$ x-ray diagnostics described above, vacuum fast electron energy spectra were measured with a magnetic deflection spectrometer ${ }^{10}$ at $44^{\circ}$ from the laser axis in the horizontal plane.
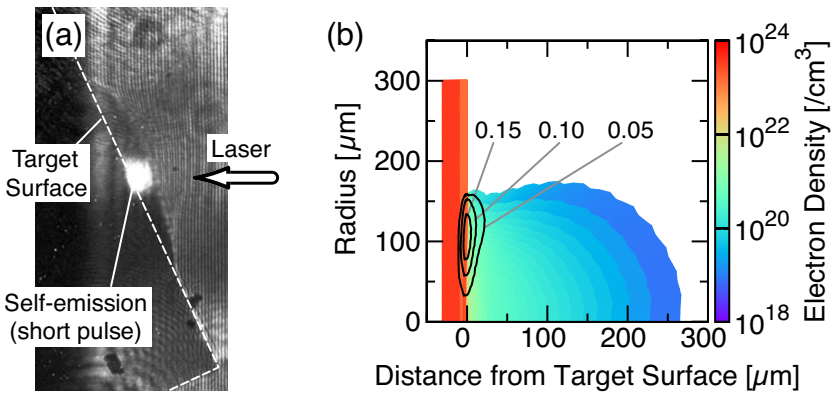

(c)

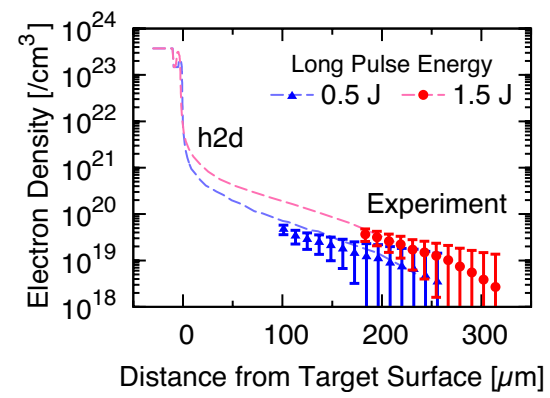

FIG. 2. (Color online) (a) Interferogram of plasma produced by the $0.5 \mathrm{~J}$ long pulse beam. Dashed line represents the target position. (b) Preplasma density profile due to $0.5 \mathrm{~J}$ long pulse irradiation as simulated by $\mathrm{h} 2 \mathrm{~d}$ at the short pulse injection timing. The contour plots of the thermoelectric magnetic field are also shown. The numbers indicate the magnetic field strength in units of megagauss. The field direction is into the paper. (c) Lineout of the density profile (normal to the target surface).

A typical interferogram taken with $0.5 \mathrm{~J}$ long pulse beam is shown in Fig. 2(a). The laser pulses come from the right hand side in this figure. The interferograms were Abel inverted to extract information about the density. A cylindrically symmetric plasma was assumed due to a large long pulse laser spot. The self-emission from the target due to the short pulse is evident in the interferogram due to a long exposure time of the CCD camera. The lineouts of the density profile due to the long pulse irradiation with energies of 0.5 and $1.5 \mathrm{~J}$ are shown in Fig. 2(c). The profiles were taken in the normal direction of the target surface. The spatial resolution of the interferogram was $4 \mu \mathrm{m}$ and the electron density uncertainty was $1 \times 10^{19} \mathrm{~cm}^{-3}$. Fringe shifts were not found when only the short pulse was incident onto the target.

The radiation hydrodynamics code $\mathrm{h} 2 \mathrm{~d}$ was used to simulate preplasma production. h2d is a Lagrangian, 2D radiation hydrodynamics code. ${ }^{11} \mathrm{~h} 2 \mathrm{~d}$ includes magnetohydrodynamics; therefore, the code can self-consistently simulate the profile of thermoelectric magnetic fields excited by the $\nabla n \times \nabla T$ mechanism. Figure 2(b) shows the plasma density profiles at the time of the short pulse injection, as well as the contour plot of the thermoelectric magnetic field in the case of $0.5 \mathrm{~J}$ in the long pulse beam. The estimated maximum magnetic field strength is $0.17 \mathrm{MG}$. The density profiles at two long pulse beam energies estimated with h2d are shown in Fig. 2(c), which show a good agreement with the experimental observation. The $1 / e$ lengths of the preplasma density profile are estimated at the critical density of the short pulse to be 9 and $17 \mu \mathrm{m}$ for 0.5 and $1.5 \mathrm{~J}$ long pulse energies, respectively.

Three main observations have been made: First, it was observed that the vacuum electron spectra were affected by 

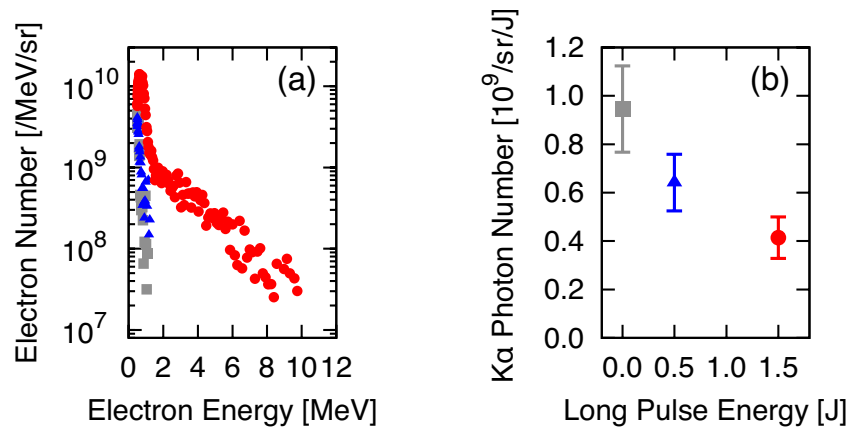

FIG. 3. (Color online) (a) Fast electron energy spectra measured in vacuum. (b) $K \alpha$ signal intensity measured with HOPG spectrometer. (ם), ( $\mathbf{\Delta}$ ), and ( ) are for the cases of without long pulse, $0.5 \mathrm{~J}$ pulse, and $1.5 \mathrm{~J}$ pulse, respectively.

the preplasma level. The spectra show a clear difference when a large preplasma is created, as shown in Fig. 3(a). The maximum energy was $10 \mathrm{MeV}$ with $1.5 \mathrm{~J}$ long pulse beam, while it was about $1 \mathrm{MeV}$ with the intrinsic prepulse. High temperature components $(2.6 \mathrm{MeV})$ with energies above $3 \mathrm{MeV}$ were only observed with the long pulse beam $(1.5 \mathrm{~J})$. Note that there was no electron signal on the spectrometer when the shot was taken with only the long pulse beam. It should be also pointed out that the low energy electrons below $2 \mathrm{MeV}$ have a similar temperature $(0.2 \mathrm{MeV})$ for all plasma profiles. This is consistent with the Wilks ${ }^{12}$ and Beg ${ }^{13}$ scalings, which predict about $0.3 \mathrm{MeV}$ temperature at the intensity. The results of the high energy electrons tail are consistent with the recent modeling, ${ }^{14,15}$ where the fast electron energy spectra are shown to be related to the preplasma scale length. In the case of a large preplasma, the electrons are accelerated through a long distance in the underdense preplasma and gain higher energies. More details are given in Refs. 14 and 15. In addition, the laser power (30 TW) is much higher than the threshold power for relativistic selffocusing $\left(17.4 n_{\mathrm{c}} / n_{\mathrm{e}} \mathrm{GW}\right.$, where $n_{\mathrm{e}}$ and $n_{\mathrm{c}}$ are the background plasma density and the critical density, respectively) ${ }^{16}$ in low density plasmas. Such a self-focused laser pulse will have higher intensities; therefore high temperature components of fast electrons can be produced. The tail was only observed in the $1.5 \mathrm{~J}$ case but not in the $0.5 \mathrm{~J}$ case because of the differences in the preplasma scale length by a factor of 2 .

Second, the preplasma effect was clearly seen on the total $K \alpha$ x-ray yield. The $\mathrm{Cu} K \alpha$ yields observed with the HOPG spectrometer are shown as a function of the long pulse energy in Fig. 3(b). The signal was reduced by up to $60 \%$ with a large preplasma. The signal reduction could be explained by the fast electrons stopping within the preplasma, namely, due to the bottlenecking by the self-excited fields in the low density plasmas. ${ }^{6}$ As discussed above, the fast electron generation process as well as the conversion efficiency could be changed by the preplasma. It is worth mentioning that the electron numbers observed with the electron spectrometer may not be relevant to the electron numbers inside the target because of the electron beam divergence and the effect of self-excited fields around the target. ${ }^{17}$ A detailed investigation of the physics that reduces that yield
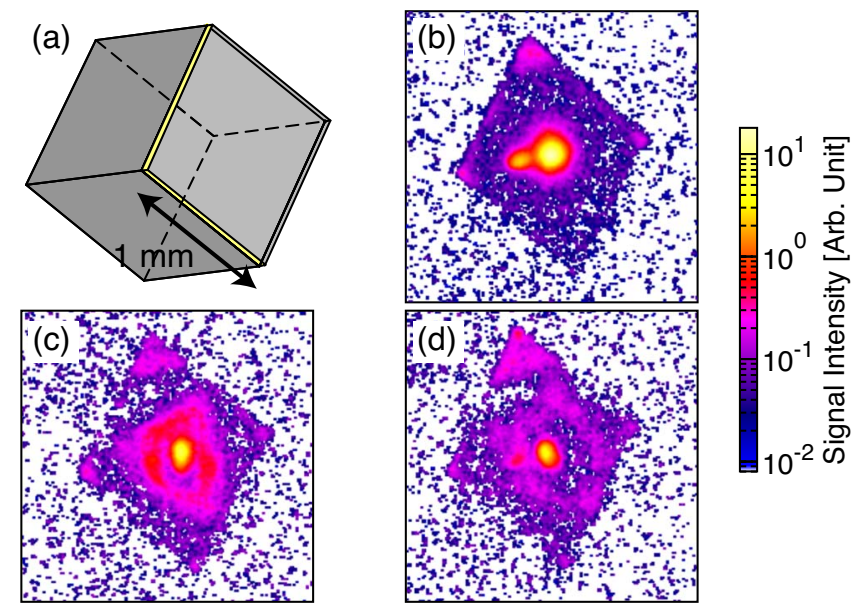

(e)

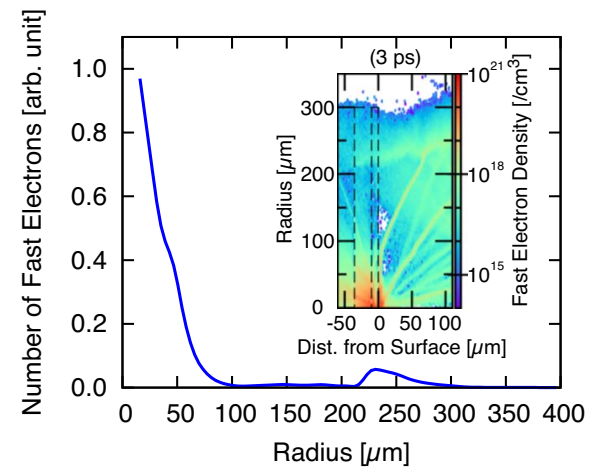

FIG. 4. (Color online) (a) Target view from the $K \alpha$ imager. The laser pulses come from the right hand side in this view. Typical $K \alpha$ images (b) without the long pulse, (c) with $0.5 \mathrm{~J}$, and (d) with $1.5 \mathrm{~J}$ in the long pulse. (e) Time-integrated fast electron number across the $\mathrm{Cu}$ layer simulated with LSP code. The inset shows fast electron density at $3.0 \mathrm{ps}$. In the inset, the electron beam is injected from the right side to the target indicated by dashed lines $(10 \mu \mathrm{m} \mathrm{Al} / 25 \mu \mathrm{m} \mathrm{Cu} /$ rear Al).

is out of the scope of this letter. It will be investigated in the future with numerical modeling including laser-plasma interactions.

Third, the spatial distribution of $K \alpha \mathrm{x}$-ray emission was monitored with the Bragg crystal imager, as shown in Figs. 4(b)-4(d) with various preplasma levels. The target view from the crystal is shown in Fig. 4(a). It can be seen that an intense small $K \alpha$ spot is observed as the result of only the short pulse target interaction. When a long laser pulse was used to create preplasma, the brightness of the central spot was reduced, which is consistent with the HOPG spectrometer results. It should be noted that a small spot seen on the left hand side of the central bright spot in Fig. 4(b) is caused by the focusing pattern of the short pulse. A ring structure was found around the central spot only when the preplasma was present, as shown in Figs. 4(c) and 4(d). The ring size is estimated at about 450-500 $\mu \mathrm{m}$ in diameter with the long pulse laser energy of $0.5 \mathrm{~J}$. The ring structure is weaker and has a larger diameter in the case of $1.5 \mathrm{~J}$. X-ray ring structures were observed by Amiranoff et al. ${ }^{18}$ at the laser intensities of $10^{15-16} \mathrm{~W} / \mathrm{cm}^{2}$ and Koch et al. ${ }^{19}$ at $10^{19} \mathrm{~W} / \mathrm{cm}^{2}$. In the first case, the lateral transport of a surface current of fast electrons caused the ring structure. In the second case, the Weibel instability was cited as the reason for the ring structure. The central spot was not observed in the experiments 
mentioned above. It is unlikely that the above mentioned mechanisms are responsible for our results for the following reasons: the fluorescence $\mathrm{Cu}$ layer was coated by a solid $\mathrm{Al}$ layer, therefore the surface current should not create the $K \alpha$ $\mathrm{x}$ rays. Also, the ring structure was seen around a bright central spot in our experiment, which is not consistent with arguments in Ref. 19.

In order to understand the mechanism for the creation of the ring structure of $K \alpha \mathrm{x}$ rays with a large scale preplasma, the hybrid PIC simulation code LSP (Ref. 20) was used to model electron transport in the presence of large preformed plasmas. In the LSP calculations, the preplasma profile including the thermoelectric magnetic field from the $\mathrm{h} 2 \mathrm{~d}$ modeling ( $0.5 \mathrm{~J}$ long pulse case) was used as the initial condition of the plasma. As is typical for Lagrangian hydrodynamics codes, the density profile produced by $\mathrm{h} 2 \mathrm{~d}$ has a very steep plasma-vacuum boundary at the first zone. The boundary was smoothed with extrapolated plasma that had a similar scale length with the modeled plasma to prevent any numerical phenomena occurring at the boundary. Fast electrons (20-50 $\mu \mathrm{m}$ spot with a half divergence angle of $30^{\circ}, 0.5 \mathrm{ps}$ pulse duration) were injected in the preplasma toward the solid target at three times of the relativistic critical density. The fast electron energy spectrum was independent of the direction of electron injection and given by the Maxwellian function with a temperature of $0.3 \mathrm{MeV}$. Figure 4(e) shows the time-integrated fast electron number profile taken along the transverse direction in the $\mathrm{Cu}$ layer. The fast electron density map at 3 ps is shown in the inset of Fig. 4(e). An increase in the accumulated fast electron number is seen at around 200-250 $\mu \mathrm{m}$ away from the central peak in the profile. The position of this bump in electron number agrees very well with the size of the ring structure of the $K \alpha$ x rays. The mechanism for the creation of the ring structure is the following: a filament of electrons with low energies is deflected by the thermoelectric magnetic field in the preplasma. The deflected electrons excite an electrostatic field at around $80 \mu \mathrm{m}$ from the solid surface where the background plasma density is comparable to the fast electron density (of the order of $10^{16-17} / \mathrm{cm}^{3}$ ). This electrostatic field deflects the electron beam along the density contour so that the fast electrons are contained in the plasma. The flow of electrons headed to the target enters the $\mathrm{Cu}$ layer far away from the central region as seen in Fig. 4(e). The ring size is therefore dependent on the density and field spatial profiles of the preplasma. In addition to this, the fast electron density, which is a function of the laser intensity, can change the ring structure. Such a structure was not seen if the preplasma was not present in LSP calculations, which is consistent with the experimental data taken without the long pulse beam.

In summary, the fast electron production and transport have been investigated at the short pulse laser intensity of $2 \times 10^{18} \mathrm{~W} / \mathrm{cm}^{2}$ with a preplasma created by a separate long pulse beam with energies up to $1.5 \mathrm{~J}$. The fast electrons observed in vacuum had high temperature components with a large preplasma. The $K \alpha$ photons emitted from the fluorescence layer of the target were reduced significantly due to the existence of preplasma. A ring structure of $\mathrm{x}$ rays is observed when a large scale preplasma is produced. The hybrid PIC simulations show that the fast electrons are affected by the fields during their transport in the preplasma and enter the target at the position where the ring structure was observed in the experiment. Preplasma effects should be studied with a FI relevant intensity laser pulse $\left(\sim 10^{20} \mathrm{~W} / \mathrm{cm}^{2}\right)$.

The authors acknowledge the GMII laser technical staff for the excellent support and Dr. M. H. Key and Professor S. Krasheninnikov for useful discussions.

This work was supported by the U.S. DOE under Contract Nos. DE-FG02-05ER54834 (ACE) and DE-FC0204ER54789 (Fusion Science Center).

${ }^{1}$ M. Tabak, J. Hammer, M. E. Glinsky, W. L. Kruer, S. C. Wilks, J. Woodworth, E. M. Campbell, M. D. Perry, and R. J. Mason, Phys. Plasmas 1, 1626 (1994).

${ }^{2}$ R. Kodama, H. Shiraga, K. Shigemori, Y. Toyama, S. Fujioka, H. Azechi, H. Fujita, H. Habara, T. Hall, Y. Izawa, T. Jitsuno, Y. Kitagawa, K. M. Krushelnick, K. L. Lancaster, K. Mima, K. Nagai, M. Nakai, H. Nishimura, T. Norimatsu, P. A. Norreys, S. Sakabe, K. A. Tanaka, A. Youssef, M. Zepf, and T. Yamanaka, Nature (London) 418, 933 (2002).

${ }^{3}$ A. G. MacPhee, L. Divol, A. J. Kemp, K. U. Akli, F. N. Beg, C. D. Chen, H. Chen, D. S. Hey, R. J. Fedosejevs, R. R. Freeman, M. Henesian, M. H. Key, S. Le Pape, A. Link, T. Ma, A. J. Mackinnon, V. M. Ovchinnikov, P. K. Patel, T. W. Phillips, R. B. Stephens, M. Tabak, R. Town, Y. Y. Tsui, L. D. Van Woerkom, M. S. Wei, and S. C. Wilks, Phys. Rev. Lett. 104, 055002 (2010).

${ }^{4}$ S. Wilks, P. E. Young, J. Hammer, M. Tabak, and W. L. Kruer, Phys. Rev. Lett. 73, 2994 (1994).

${ }^{5}$ A. Pukhov and J. Meyer-ter-Vehn, Phys. Rev. Lett. 76, 3975 (1996).

${ }^{6}$ M. S. Wei, A. A. Solodov, J. Pasley, R. B. Stephens, D. R. Welch, and F. N. Beg, Phys. Plasmas 15, 083101 (2008).

${ }^{7}$ W. Theobald, Bull. Am. Phys. Soc. 54, 187 (2009).

${ }^{8}$ H. Habara, G. Xu, T. Jitsuno, R. Kodama, K. Suzuki, K. Sawai, C. P. J. Barty, T. Kawasaki, H. Kitamura, K. Kondo, K. Mima, N. Miyanaga, Y. Nakata, H. Shiraga, K. A. Tanaka, K. Tsubakimoto, and M. C. Rushford, J. Phys.: Conf. Ser. 112, 032017 (2008).

${ }^{9}$ X. Llovet, C. Merlet, and F. Salvat, J. Phys. B 33, 3761 (2000).

${ }^{10}$ K. A. Tanaka, T. Yabuuchi, T. Sato, R. Kodama, Y. Kitagawa, T. Takahashi, T. Ikeda, Y. Honda, and S. Okuda, Rev. Sci. Instrum. 76, 013507 (2005)

${ }^{11} \mathrm{~h} 2 \mathrm{~d}$ is the 2D version of HYADES; J. T. Larsen and S. Lane, J. Quant. Spectrosc. Radiat. Transf. 51, 179 (1994).

${ }^{12}$ S. C. Wilks, W. L. Kruer, M. Tabak, and A. B. Langdon, Phys. Rev. Lett. 69, 1383 (1992).

${ }^{13}$ F. N. Beg, A. R. Bell, A. E. Dangor, C. N. Danson, A. P. Fews, M. E. Glinsky, B. A. Hammel, P. Lee, P. A. Norreys, and M. Tatarakis, Phys. Plasmas 4, 447 (1997).

${ }^{14}$ A. J. Kemp, Y. Sentoku, and M. Tabak, Phys. Rev. Lett. 101, 075004 (2008).

${ }^{15}$ H. Cai, K. Mima, A. Sunahara, T. Johzaki, H. Nagatomo, S. Zhu, and X. T. He, Phys. Plasmas 17, 023106 (2010).

${ }^{16}$ P. Sprangle, C.-M. Tang, and E. Esarey, IEEE Trans. Plasma Sci. 15, 145 (1987).

${ }^{17}$ T. Yabuuchi, K. Adumi, H. Habara, R. Kodama, K. Kondo, T. Tanimoto, K. A. Tanaka, Y. Sentoku, T. Matsuoka, Z. L. Chen, M. Tampo, A. L. Lei, and K. Mima, Phys. Plasmas 14, 040706 (2007).

${ }^{18}$ F. Amiranoff, K. Eidmann, R. Sigel, R. Fedosejevs, A. Maaswinkel, Y. lu Teng, J. D. Kilkenny, J. D. Hares, D. K. Bradley, B. J. MacGowan, and T. J. Goldsack, J. Phys. D: Appl. Phys. 15, 2463 (1982).

${ }^{19}$ J. A. Koch, M. H. Key, R. R. Freeman, S. P. Hatchett, R. W. Lee, D. Pennington, R. B. Stephens, and M. Tabak, Phys. Rev. E 65, 016410 (2001).

${ }^{20}$ D. R. Welch, D. V. Rose, M. E. Cuneo, R. B. Campbell, and T. A. Mehlhorn, Phys. Plasmas 13, 063105 (2006). 